\title{
Recurrent vertebral fractures in a young adult: a closer look at bone health in type 1 diabetes mellitus
}

\author{
Eleanor P Thong1,2, Sarah Catford1,3, Julie Fletcher ${ }^{4}$, Phillip Wong1,3, Peter J Fuller1,3, Helena Teede ${ }^{1,2}$ and \\ Frances Milat 1,3
}

1Department of Endocrinology, Monash Health, Clayton, Australia, ${ }^{2}$ Monash Centre for Health Research and Implementation, Clayton, Australia, ${ }^{3} \mathrm{Hudson}$ Institute of Medical Research, Clayton, Australia, and ${ }^{4}$ Department of Anatomical Pathology, Concord Repatriation General Hospital, Concord, Australia

Correspondence should be addressed to F Milat

Email

fran.milat@hudson.org.au

\section{Summary}

The association between type 1 diabetes mellitus (T1DM) and bone health has garnered interest over the years. Fracture risk is known to be increased in individuals with T1DM, although bone health assessment is not often performed in the clinical setting. We describe the case of a 21-year-old male with longstanding T1DM with multilevel vertebral fractures on imaging, after presenting with acute back pain without apparent trauma. Dual-energy X-ray absorptiometry (DXA) revealed significantly reduced bone mineral density at the lumbar spine and femoral neck. Extensive investigations for other secondary or genetic causes of osteoporosis were unremarkable, apart from moderate vitamin D deficiency. High-resolution peripheral quantitative computed tomography and bone biospy revealed significant alterations of trabecular bone microarchitecture. It later transpired that the patient had sustained vertebral fractures secondary to unrecognised nocturnal hypoglycaemic seizures. Intravenous zoledronic acid was administered for secondary fracture prevention. Despite anti-resorptive therapy, the patient sustained a new vertebral fracture after experiencing another hypoglycaemic seizure in his sleep. Bone health in T1DM is complex and not well understood. There are significant challenges in the assessment and management of osteoporosis in T1DM, particularly in young adults, where fracture prediction tools have not been validated. Clinicians should be aware of hypoglycaemia as a significant risk factor for fracture in patients with T1DM.

\section{Learning points:}

- Type 1 diabetes mellitus (T1DM) is a secondary cause of osteoporosis, characterised by reduced bone mass and disturbed bone microarchitecture.

- Hypoglycaemic seizures generate sufficient compression forces along the thoracic column and can cause fractures in individuals with compromised bone quality.

- Unrecognised hypoglycaemic seizures should be considered in patients with T1DM presenting with fractures without a history of trauma.

- Patients with T1DM have increased fracture risk and risk factors should be addressed. Evaluation of bone microarchitecture may provide further insights into mechanisms of fracture in T1DM.

- Further research is needed to guide the optimal screening and management of bone health in patients with T1DM. 


\section{Background}

Type 1 diabetes mellitus (T1DM) is an immune-mediated condition culminating in the destruction of pancreatic beta cells, which are necessary for insulin production. T1DM is commonly diagnosed in childhood and young adults, thus reflecting the burden of this chronic disease in a young population, who are ultimately at risk of the long-term complications of diabetes. In recent years, there has been growing interest and awareness of the pathophysiology and mechanisms behind diabetic bone disease. Although T1DM is an established risk factor for osteoporosis and fracture, bone health in patients with T1DM is not routinely assessed. This may be in part due to the lack of guidelines for fracture risk assessment and management of such patients, particularly in children and young adults. We present the challenges in diagnosis and management of recurrent fractures secondary to hypoglycaemic seizures in a patient with T1DM.

\section{Case presentation}

A 21-year-old male Caucasian university student presented to the emergency department after awaking with suddenonset, severe inter-scapular back pain. Multiple thoracic vertebral fractures were initially appreciated on plain X-ray films, and subsequent magnetic resonance imaging (MRI) confirmed widespread acute and subacute compression fractures throughout the thoracic and lumbar spine at T2-5, T9 and T10, L4 and L5 (Fig. 1). The patient reported no antecedent trauma or recent falls.

His history was significant for a 12-year duration of T1DM, which was managed with a basal-bolus insulin regimen, under the care of an endocrinologist. He reported good glycaemic control historically and self-monitoring of blood sugar levels several times a day with infrequent hypoglycaemic episodes. He was a non-smoker and only consumed alcohol socially. Dietary calcium intake was adequate, averaging three servings daily. There was no history of fractures in childhood, and no personal or
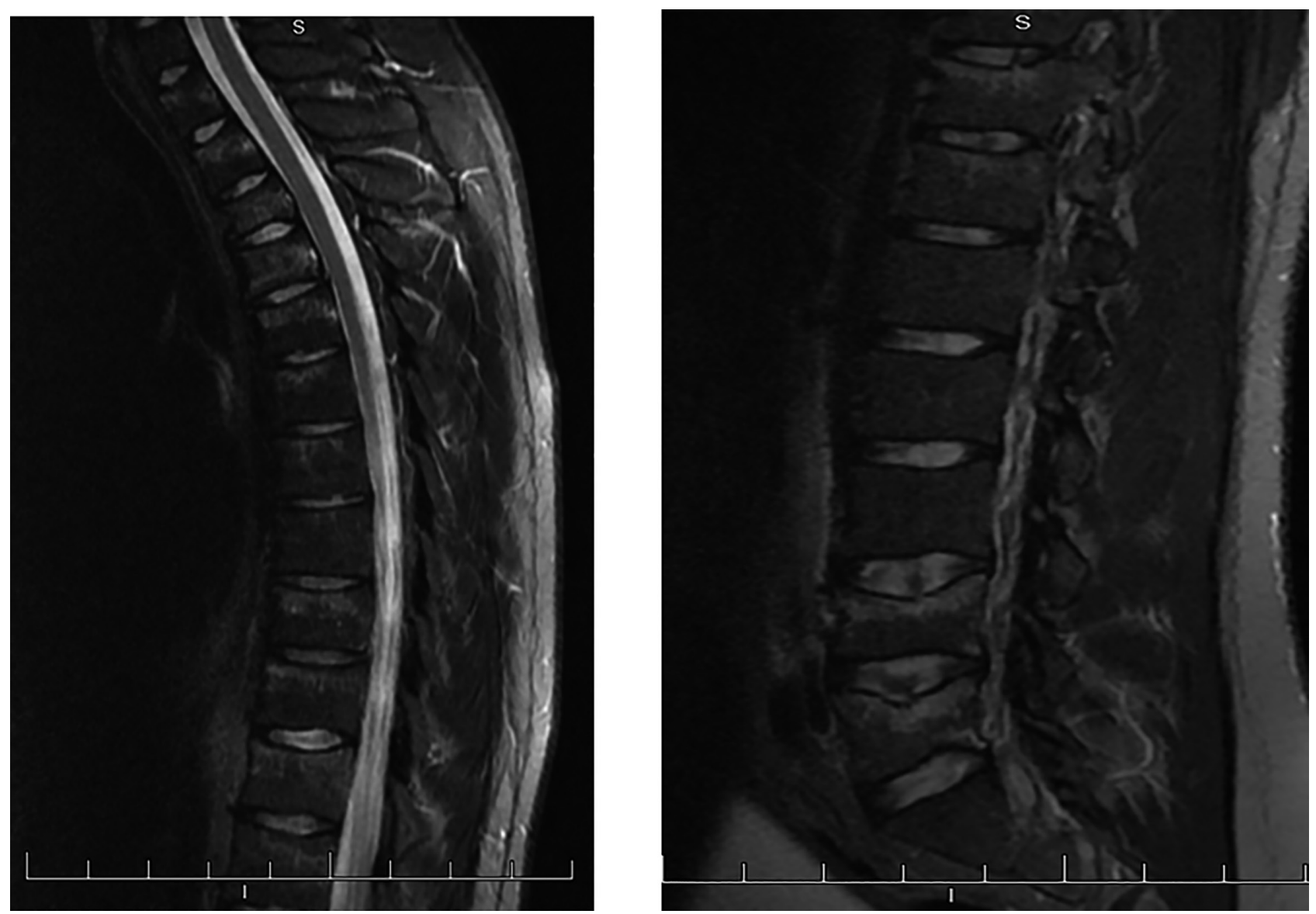

Figure 1

T2-sagittal MRI demonstrating hyperintense marrow signal abnormalities in several vertebral compression fractures. MRI, magnetic resonance imaging. 
family history of bone disease or minimal trauma fractures. Apart from multiple daily injections of insulin detemir and aspart, he reported no other regular medications or previous glucocorticoid use. Clinical examination revealed a well-virilised male with normal testicular volumes, and a height and weight of $181 \mathrm{~cm}$ and $94 \mathrm{~kg}$ respectively. Fundoscopy revealed no evidence of retinopathy, and neurological examination was unremarkable; there was no evidence of blue sclerae or kyphosis.

\section{Investigation}

Initial laboratory investigations revealed a normal electrolyte profile and renal function, full blood count and thyroid function tests. He had a glycated haemoglobin of $7.8 \%(62 \mathrm{mmol} / \mathrm{L})$ and had no evidence of urinary microalbuminuria. Moderate vitamin D deficiency $(28 \mathrm{nmol} / \mathrm{L})$ was identified; however, corrected calcium, phosphate and parathyroid hormone levels were all within reference limits. Coeliac disease antibodies were negative, as were other investigations for secondary osteoporosis, including Cushing's syndrome, hyperthyroidism, hypogonadism, liver disease, mastocystosis, idiopathic hypercalciuria and multiple myeloma (Table 1). Boneturnover markers (C-terminal telopeptide (CTX) and procollagen type 1 amino-terminal propeptide (P1NP)) were elevated, in keeping with the increased bone-turnover state in the context of his recent fractures. Bone mineral density on DXA was severely reduced, with $Z$-scores of -2.9 at L2-L3 and -3.0 at the left femoral neck respectively.

Four months later, the patient developed new acute fractures at T7 and L2, after a witnessed hypoglycaemic seizure at home. During his inpatient admission, he was noted to have multiple nocturnal hypoglycaemic episodes with impaired awareness of hypoglycaemia. Investigations for a primary seizure disorder with MRI brain and electroencephalogram were unremarkable.

Given the extensive fracture history, a bone marrow aspirate and trephine (with tetracycline-labelled bone biopsy) of the posterior iliac crest was performed to exclude a malignant cause. No haematological or malignant disease was identified, but disruption to the bone trabecular architecture was evident in cancellous bone, characterised by a reduction in trabecular number, thinning and increased separation of trabeculae (Fig. 2). There were no features of osteomalacia, indicated by normal tetracycline uptake at the bone-mineralising surfaces. High-resolution peripheral quantitative computed tomography (HR-pQCT) was also performed of the radius and tibia, revealing significant cortical and
Table 1 Summary of biochemical investigations and HR-pQCT parameters.

\begin{tabular}{|c|c|c|}
\hline & Value & RR or centile* \\
\hline \multicolumn{3}{|l|}{ Biochemical investigations } \\
\hline HbA1c (\%) & $7.8 \%$ & $\leq 6.0$ \\
\hline Creatinine $(\mu \mathrm{mol} / \mathrm{L})$ & 96 & $60-105$ \\
\hline Urea $(\mathrm{mmol} / \mathrm{L})$ & 4.4 & $4.4-9.0$ \\
\hline Estimated GFR (mL/min/1.73 $\left.\mathrm{m}^{2}\right)$ & $>90$ & $>90$ \\
\hline Alkaline phosphatase (U/L) & 136 & $42-135$ \\
\hline Corrected calcium (mmol/L) & 2.49 & $2.14-2.50$ \\
\hline Magnesium (mmol/L) & 0.90 & $0.66-1.07$ \\
\hline Phosphate $(\mathrm{mmol} / \mathrm{L})$ & 0.65 & $0.60-1.30$ \\
\hline Parathyroid hormone (pmol/L) & 4.5 & $1.6-6.9$ \\
\hline Vitamin D (nmol/L) & 28 & $>50$ \\
\hline $\mathrm{TSH}(\mathrm{mU} / \mathrm{L})$ & 1.60 & $0.3-5.00$ \\
\hline $\mathrm{FSH}(\mathrm{U} / \mathrm{L})$ & 2.8 & $1.4-13.6$ \\
\hline Luteinising hormone (U/L) & 1.0 & $0.6-12.1$ \\
\hline Total testosterone $(\mathrm{nmol} / \mathrm{L})$ & 20.6 & $8.0-30.0$ \\
\hline Prolactin (U/L) & 218 & 73-306 \\
\hline Cortisol, post ODST (nmol/L) & 42 & $<50$ \\
\hline Transglutaminase $A B(\mathrm{U} / \mathrm{mL})$ & 0 & $0-4$ \\
\hline Gliadin IgA (units) & 5 & 0-19 \\
\hline Gliadin IgG (units) & 2 & $0-19$ \\
\hline Tryptase ( $\mu \mathrm{g} / \mathrm{L})$ & 4.1 & $0.0-11.4$ \\
\hline Serum protein electrophoresis & NPD & \\
\hline CTX (ng/L) & 1435 & $400-900$ \\
\hline P1NP $(\mu \mathrm{g} / \mathrm{L})$ & 151 & $15-115$ \\
\hline \multicolumn{3}{|l|}{ HR-pQCT) parameters } \\
\hline \multicolumn{3}{|l|}{ Radius } \\
\hline Total BMD (mg/HA/cm³) & 274.2 & $<10$ th \\
\hline Cortical BMD (mg/HA/cm³) & 792.3 & $<10$ th \\
\hline Trabecular BMD (mg/HA/cm³) & 177.0 & $<25$ th \\
\hline Trabecular number $(1 / \mathrm{mm})$ & 1.67 & $<10$ th \\
\hline Trabecular thickness (mm) & 0.088 & 75 th \\
\hline Trabecular separation (mm) & 0.511 & 90th \\
\hline Cortical thickness (mm) & 0.62 & $<10$ th \\
\hline \multicolumn{3}{|l|}{ Tibia } \\
\hline Total BMD (mg/HA/cm³) & 246.3 & $<10$ th \\
\hline Cortical BMD (mg/HA/cm³) & 835.0 & $<10$ th \\
\hline Trabecular BMD (mg/HA/cm³) & 172.1 & $<10$ th \\
\hline Trabecular number $(1 / \mathrm{mm})$ & 1.87 & 25 th \\
\hline Trabecular thickness (mm) & 0.077 & 10th \\
\hline Trabecular separation (mm) & 0.459 & 75 th \\
\hline Cortical thickness (mm) & 0.82 & $<10$ th \\
\hline
\end{tabular}

Abnormal values are denoted in bold.

* $R R$ is presented for biochemical investigations and centiles for HR-pQCT parameters. Centiles are derived from age-, sex- and site-specific centile curves for HR-pQCT parameters using a Canadian reference population from the study by Burt et al. (1).

BMD, bone mineral density; CTX, C-terminal telopeptide; GFR, glomerular filtration rate; $\mathrm{HA}$, hydroxyapatite; HR-pQCT, high-resolution peripheral quantitative computed tomography; NPD, no paraprotein detected; ODST, overnight dexamethasone suppression test; P1NP, N-terminal propeptide of type 1 collagen; RR, reference range; $\mathrm{HbA1c}$, glycated haemoglobin; TSH, thyroid stimulating hormone; $\mathrm{FSH}$, follicle stimulating hormone; $A B$, antibodies.

trabecular deficits. In particular, his cortical and trabecular volumetric bone mineral density, and cortical thickness, were under the 10th centile of age-, sex- and site-matched 

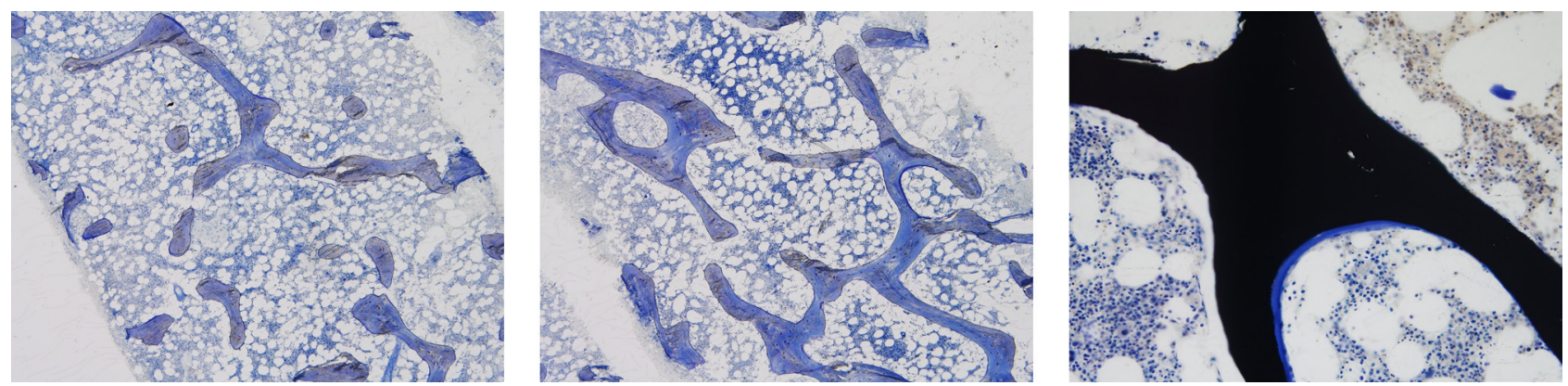

\section{Figure 2}

(A) Toluidine blue stain at $\times 40$ showing reduced numbers, abnormal shapes, thinning and reduced connectivity of the bony trabeculae. (B) Toluidine blue stain at $\times 40$ showing an area of relatively normal trabecular bone formation in the patient. (C) Von Kossa stain for calcium at $\times 100$ showing a normal small smooth segment of osteoid on the surface of normally calcified bone.

normative values derived from a reference population of adults (1) (Table 1). A clinical geneticist reviewed the patient, and no hereditary cause of bone disease was identified. Therefore, the working diagnosis was extensive vertebral fractures secondary to unrecognised hypoglycaemic seizures, on a background of impaired bone microarchitecture.

\section{Treatment}

His initial management consisted of non-opioid analgesia, vitamin $\mathrm{D}$ replacement and insulin optimisation to prevent further episodes of hypoglycaemia. Zoledronic acid (5 mg) was administered intravenously for fracture prevention after he was vitamin $\mathrm{D}$ replete.

\section{Outcome and follow-up}

Fourteen months later, the patient re-presented with back pain, following another hypoglycaemic seizure, which occurred in his sleep. Repeat spine imaging revealed a new fracture of the L1 vertebra. Given the recurrent fracture despite anti-resorptive therapy, consideration was given to teriparatide, an anabolic agent, for the treatment of his severe osteoporosis. Continuous glucose monitoring was also organised to better delineate his nocturnal glycaemic pattern, in a bid to reduce further hypoglycaemic episodes.

\section{Discussion}

The link between bone health and T1DM has garnered increasing attention in recent years. Meta-analyses of observational studies have reported a threefold to sevenfold increased risk of hip fracture in individuals with T1DM, compared to controls $(2,3)$. T1DM is an established cause of secondary osteoporosis and the pathogenesis of fracture in this population appears to be multifactorial (Table 2). T1DM is characterised by a state of hypoinsulinaemia and insulin-like growth factor (IGF-1) deficiency. This has been postulated to impair osteoblast function, giving rise to a low bone-turnover state $(4,5,6)$. Peak bone mass is acquired at puberty and accrued by the third decade of life. The development of T1DM in childhood may interfere with peak bone mass accrual, such that individuals may have reduced bone mineral density (BMD) in adulthood. Advanced glycation end products (AGEs) from chronic hyperglycaemia can

Table 2 Mechanisms of fracture in individuals with type 1 diabetes mellitus.

Reduced bone mass

- Insulin/IGF-1 deficiency

- Hypogonadism

- Nephropathy (CKD-MBD)

- Concomitant autoimmunity: Graves' and coeliac disease

- Failure to achieve peak bone mass (childhood-onset T1DM) Impaired bone quality and biomechanical properties

- Disrupted trabecular bone architecture

- Altered cortical bone geometry

- Hyperglycaemia: altered bone collagen matrix by AGEs Increased risk of falls and trauma

- Neuropathy

- Retinopathy

- Hypoglycaemia

- Amputations

Other

- Vitamin D deficiency

- Chronic inflammation

- Family history of osteoporosis

- Smoking

- Genetic bone disorder, e.g. OI

- Corticosteroid use

AGEs, advanced glycation end products; CKD-MBD, chronic kidney disease mineral and bone disorder; IGF-1, insulin-like growth factor; OI, osteogenesis imperfecta. 
disrupt collagen matrix and impact negatively on bone biomechanical properties $(5,6,7)$. Altered bone mineral metabolism is often seen with diabetic chronic kidney disease, and concomitant autoimmunity, such as thyroid and coeliac disease, may contribute to further loss of bone mass. Finally, the long-term sequelae of diabetes, including retinopathy, neuropathy and hypoglycaemia may contribute to reduced physical function and increased propensity to falls (4).

Hypoglycaemic seizures, such as in our patient, have been implicated in multilevel vertebral fractures in a few case reports $(8,9,10)$. Compressive forces along the anterior and middle columns of the mid-thoracic kyphotic curve during seizure activity can lead to a unique dispersion of 'flexion fractures' in the upper to mid-thoracic vertebrae (8). These fractures are usually not clinically apparent; however, the reported prevalence of seizure-associated asymptomatic fracture is as high as $15-16 \%(8,9)$.

Clinical risk factors associated with fracture in patients with T1DM have not been well defined, although several studies have reported a consistent association of microvascular complications with increased fracture risk (5). Although BMD is typically used to predict fracture risk in the general population, it may underestimate fracture risk in patients with T1DM, especially in young adults in whom the use of fracture prediction tools has not been validated (11). Bone microarchitecture is an important determinant of bone quality and strength. Histomorphometric analysis is the gold standard for the evaluation of tissue-level bone activity (12); however, it is invasive and studies examining bone histology in humans with T1DM are limited. Novel imaging modalities such as trabecular bone score and HR-pQCT can provide indirect assessments of bone microarchitecture, which may be a useful adjunct to DXA, in the assessment of bone health in patients with T1DM.

Shanbhougue et al. (13) demonstrated deficits in HR-pQCT-derived trabecular microarchitecture parameters at the ultradistal radius and tibia in adults with T1DM and microvascular disease, compared to ageand gender-matched non-diabetic controls. Our patient exhibited a similar pattern of bone microarchitecture disturbance on HR-pQCT, and bone histomorphometric findings were consistent with that observed in murine models of T1DM $(14,15)$.

This case study demonstrates recurrent fractures secondary to trauma from hypoglycaemic seizures underpinned by impaired bone microarchitecture, in a young adult with T1DM, with no other secondary causes of accelerated bone loss. While our patient was young, exhibited reasonable glycaemic control and had no microvascular complications, we postulate that the development of T1DM in early childhood may have had significant detrimental impacts on peak bone mass accrual and bone microstructure. To our knowledge, this is the first report correlating HR-pQCT and histomorphometric evidence of dysfunctional bone microarchitecture in a fracturing patient with T1DM.

Despite treatment with intravenous bisphosphonate, the patient sustained a new vertebral fracture after another hypoglycaemic seizure. This raises the importance of hypoglycaemia prevention in T1DM, particularly those with established osteoporosis. Recurrent hypoglycaemic episodes can diminish the sympathoadrenal response and lower the threshold at which symptoms occur (16). Patients with impaired awareness of hypoglycaemia are at risk of falls and trauma, and plain radiographs of the thoracolumbar spine should be considered to exclude occult fractures in high-risk patients, where appropriate. The use of continuous glucose monitoring sensors may aid in the detection of unrecognised nocturnal hypoglycaemia.

The optimal therapy for low BMD in young adults with T1DM has not been evaluated. Although post hoc analyses of randomised controlled trials have demonstrated similar efficacy of bisphosphonates in incrementing BMD in post-menopausal patients with type 2 diabetes mellitus and non-diabetic controls (17), this may not be applicable to a younger cohort with T1DM. Given that bone disease in T1DM is characterised by a low bone-turnover state, treatment with bisphosphonates may further suppress bone turnover in such patients. Anabolic agents, such as teriparatide, increase bone formation and may be an ideal therapy for patients with T1DM bone disease. Intermittent parathyroid hormone (PTH) therapy has been shown to increase trabecular bone volume in rodents with T1DM (18), but no such studies have been performed in humans thus far.

In conclusion, T1DM causes disruption to bone microarchitecture and matrix in several ways and can increase susceptibility to falls and trauma, from hypoglycaemia or microvascular complications (Table 2). Unrecognised hypoglycaemic seizures should be considered in patients with T1DM presenting with fractures without a history of trauma. The management of osteoporosis in a young adult with T1DM is challenging, and further research is needed to guide the optimal screening and management of bone health in T1DM. 


\section{Declaration of interest}

The authors declare no conflicts of interest that could be perceived as prejudicing the impartiality of the research reported.

\section{Funding}

This research did not receive any specific grant from any funding agency in the public, commercial or not-for-profit sector

\section{Patient consent}

Written informed consent for publication of their de-identified clinical information and clinical images was obtained from the patient.

\section{Author contribution statement}

E P T performed the literature review and wrote the manuscript; E P T, S C and $\mathrm{F} \mathrm{M}$ were involved in the care of the patient; J F was the anatomical pathologist and $\mathrm{P}$ W, $\mathrm{P} J \mathrm{~F}$ and $\mathrm{H}$ T made critical revisions. All authors approved the final version of the manuscript.

\section{References}

1 Burt LA, Liang Z, Sajobi TT, Hanley DA \& Boyd SK. Sex- and sitespecific normative data curves for HR-pQCT Journal of Bone and Mineral Research 201631 2041-2047. (https://doi.org/10.1002/ jbmr.2873)

2 Vestergaard P. Discrepancies in in bone mineral density and fracture risk in patients with type 1 and type 2 diabetes - a metaanalysis. Osteoporosis International 200718 427-444. (https://doi.org/10.1007/ s00198-006-0253-4)

3 Shah VN, Shah CS \& Snell-Burgeon JK. Type 1 diabetes and risk of fracture: meta-analysis and review of the literature. Diabetic Medicine 201532 1134-1142. (https://doi.org/10.1111/dme.12734)

4 Hofbauer LC, Brueck CC, Singh SK \& Dobnig D. Osteoporosis in patients with diabetes mellitus. Journal of Bone and Mineral Research 200722 1317-1328. (https://doi.org/10.1359/jbmr.070510)

5 Hough FS, Pierroz DD, Cooper C, Ferrari SL \& IOF CSA Bone and Diabetes Working Group. Mechanisms in endocrinology: mechanisms and evaluation of bone fragility in type 1 diabetes mellitus. European Journal of Endocrinology 2014174 R127-R138. (https://doi.org/10.1530/EJE-15-0820)

6 Khan TS \& Fraser L. Type 1 diabetes and osteoporosis: from molecular pathways to bone phenotype. Journal of Osteoporosis 2015 2015 174186. (https://doi.org/10.1155/2015/174186)

7 Zhukouskaya VV, Shepelkevich AP, Dydyshko Y, Cairoli E \& Chiodini I. Bone health in type 1 diabetes: focus on evaluation and treatment in clinical practice. Journal of Endocrinological Investigation 201538 941-950. (https://doi.org/10.1007/s40618015-0284-9)

8 Takahashi T, Tominanga T, Shamoto H, Shimizu, H \& Yoshimoto T. Seizure-induced thoracic spine compression fracture: case report. Surgical Neurology 200258 214-216. (https://doi.org/10.1016/S00903019(02)00837-6)

9 McCullen GM \& Brown CC. Seizure-induced thoracic burst fractures: a case report. Spine 199419 77-79. (https://doi. org/10.1097/00007632-199401000-00017)

10 Majkowska L \& Bohatryrewicz A. Thoracic spine fracture in the course of severe nocturnal hypoglycaemia in young patients with type 1 diabetes mellitus - the role of low bone mineral density. American Journal of Emergency Medicine 201432 816.e5-816.e7. (https://doi.org/10.1016/j.ajem.2013.12.055)

11 Ferrari S, Biachi ML, Eisman JA, Foldes AJ, Adami S, Wahl DA, Stepan JJ, de Vernejoul MC, Kaufman JM \& IOF Committee of Scientific Advisors Working Group on Osteoporosis Pathophysiology. Osteoporosis in young adults: pathophysiology, diagnosis and management. Osteoporosis International 201223 2735-2748. (https:// doi.org/10.1007/s00198-012-2030-x)

12 Allen MR \& Burr DB. Techniques in histomorphometry. In Basic and Applied Bone Biology, 1st ed., ch 7, p 131. Eds DB Burr \& MR Allen. London: Academic Press, 2013.

13 Shanbhougue VV, Hansen S, Frost M, Jørgensen NR, Hermann AP, Henriksen JE \& Brixen K. Bone geometry, volumetric density, microarchitecture, and estimated bone strength assessed by HR-pQCT in adult patients with type 1 diabetes mellitus. Journal of Bone and Mineral Research 201530 2188-2199. (https://doi. org/10.1002/jbmr.2573)

14 Thrailkill KM, Liu L, Wahl EC, Bunn RC, Perrien DS, Cockrell GE, Skinner RA, Hogue WR, Carver AA, Fowlkes JL, et al. Bone formation is impaired in a model of type 1 diabetes. Diabetes $2005 \mathbf{5 4}$ 2875-2881. (https://doi.org/10.2337/diabetes.54.10.2875)

15 Silva MJ, Brodt MD, Lynch MA, McKenzie JA, Tanouye KM, Nyman JS \& Wang X. Type 1 diabetes in young rats leads to progressive trabecular bone loss, cessation of cortical bone growth, and diminished whole bone strength and fatigue life. Journal of Bone and Mineral Research 200924 1618-1627. (https://doi.org/10.1359/ jbmr.090316)

16 Seaquist ER, Anderson J, Childs B, Cryer P, Dagogo-Jack S, Fish L, Heller SR, Rodriguez H, Rosenzweig J \& Vigersky R. Hypoglycemia and diabetes: a report of a workgroup of the American Diabetes Association and the Endocrine Society. Diabetes Care 20135 1384-1395. (https://doi.org/10.2337/dc12-2480)

17 Schwartz AV. Efficacy of osteoporosis therapies in diabetic patients. Calcified Tissue International 2017100 165-173. (https://doi. org/10.1007/s00223-016-0177-8)

18 Motyl KJ, McCauley LK \& McCabe LR. Amelioration of type I diabetes-induced osteoporosis by parathyroid hormone is associated with improved osteoblast survival. Journal of Cellular Physiology 2012 227 1326-1334. (https://doi.org/10.1002/jcp.22844) 